\title{
Occupational Noise-Induced Hearing Loss (ONIHL) and Fishing
}

\author{
Lucio Maci* and Mario Tavolaro \\ INAIL (Italian Institute for insurance against industrial injuries), Italy
}

*Corresponding author: Lucio Maci, INAIL (Italian Institute for insurance against industrial injuries) Viale Oronzo Quarta 19/B, 73100 Lecce, Italy.

Received Date: June 07, 2019

Published Date: June 12, 2019

\begin{abstract}
Fishing is a working environment, which, due to the presence of engines and noisy equipment, can lead to hearing problems, especially for workers who are more exposed, such as motorists. It is emphasized that prevention, the only weapon available, is more difficult at sea.
\end{abstract}

Keywords: Occupational noise-induced hearing loss; Epidemiology; Noise; Audiometry

\section{Introduction}

Occupational noise-induced hearing loss (ONIHL) describes an acquired hearing impairment attributable to excessive workplace noise exposure. ONIHL is likely to contribute to a very high proportion of the cases of hearing loss in adults. In Italy noise deafness affects almost two thirds of fishermen [1]. The motorists and the Commanders present a greater risk of developing noiseinduced hearing loss compared to sailors [2]. These data are in agreement with those reported in studies conducted in other countries. The main source of noise inside of the boats is represented by the engines that produce high sound levels too in fishing vessels of less than $30 \mathrm{~m}$ in length [3]. A further aspect related to noise on fishing vessels is represented by exposure sometimes continuous; it is estimated that a 24-hour exposure at $85 \mathrm{~dB}(\mathrm{~A})$ matches at an exposure of 8 hours to $90 \mathrm{~dB}(\mathrm{~A})$. The high and constant exposure to noise, even during the few hours of rest on board, helps to develop not only the hearing loss but also sleep disorders and alterations of blood pressure and favors the occurrence of injuries [4]. On the boat, the exposure time cannot be reduced, but the researchers recommend that measures are taken to decrease the intensity of the noise. Besides, they ask to the workers to receive a training and ormation on the hearing health and on the raising awareness and sensitization to the noise exposure, to take regularly hearing tests and to be followed in the long term to warn the risks of the deafness further to the noise [5].

\section{Conclusion}

The relationship between fishing and deafness appears to be widespread among sea workers. It must to promote the culture of safety and health protection of workers in the maritime sector, in order to detect, analyze and study the risk factors in the fishing sector.

\section{Acknowledgement}

INAIL Brindisi and Lecce.

\section{Conflict of Interest}

No conflict of interest.

\section{References}

1. Fiorentino F, Bono G, Gancitano V, Garofalo G, Gristina M, et al. (2011) Environmental characterization of fishing areas -GSA 16 -Southern coasts of Sicily. In: Cataudella S, Spagnolo M (Edts.), The state of fishing and aquaculture in the Italian seas. Ministry of Agricultural, Food and Forestry Policies, Rome, Italy, pp. 66-72.

2. Cataudella S, Spagnolo M (2011) The state of fishing and aquaculture in the Italian seas. Ministero delle politiche agricole alimentari e forestali, Roma, Italy.

3. Commissione europea (2012) The common fisheries policy in figures. Essential statistical data-Luxembourg pp. 1-48.

4. Bolognini S (1994) Noise on fishing boats: considerations and prospectsOccupational safety and improvement of the quality of life in fishing work-International Fishing Fair.

5. (2018) INAIL Navigation Sector, National Training Center for Shipping"Training book for work safety in machine rooms on board ships", Rome. 